\section{Les neurones en marche}

\section{L’organisation des réseaux locomoteurs} de la moelle épinière mise à jour par la génétique

Julien Bouvier
Mammalian locomotor laboratory, Neuroscience Department, Karolinska Institutet, Retziusväg 8, 17177 Stockholm, Suède. julien.bouvier@ki.se
$>$ Chez tous les vertébrés, les mouvements de la marche requièrent le recrutement rythmique et séquentiel d'une multitude de muscles répartis de chaque côté du corps. La genèse du rythme ainsi que sa mise en forme en une activité nerveuse qui définit la séquence des contractions musculaires sont assurées par des neurones de la moelle épinière organisés en un réseau formant un «générateur central de rythme et de mise en forme » (ou CPG pour central pattern generator). Décrypter l'organisation fonctionnelle du CPG locomoteur et les déterminants génétiques qui contrôlent son assemblage sont des enjeux majeurs en neurosciences. Ces études établissent un schéma d'organisation liant gènes et fonctions, qui sera utile aux cliniciens pour mieux définir certaines conditions pathologiques et élaborer les thérapies dédiées à la réhabilitation des fonctions motrices.

\section{CPG locomoteur}

\section{et coordination bilatérale}

Les études récentes chez le rongeur ont amplement documenté les mécanismes à l'origine de la genèse du rythme locomoteur [1]. Des interneurones excitateurs de la moelle épinière ventrale élaborent, en réponse à une activation par les centres locomoteurs supra-spinaux, des décharges rythmiques synchrones et entretenues. À partir de ce rythme est établi un pattern, c'est-à-dire une mise en forme, une distribution séquentielle de l'activité nerveuse aux différents groupes musculaires afin d'assurer la coordination des mouvements. La coordination bilatérale, c'est-à-dire entre les membres situés à droite et à gauche du corps, est un exemple frappant de mise en forme, qui illustre le côté à la fois prédéterminé et adaptable du comportement (et donc du CPG). En effet, la plupart des espèces présentent un mode de coordination bilatérale prépondérant: le lapin se déplace par bonds conditionnés par des contractions synchrones des mêmes muscles au sein des membres droits et gauches, alors que la souris, comme la plupart des mammifères terrestres, présente au contraire des mouvements bilatéraux essentiellement alternés. Pour autant, cette dernière adopte occasionnellement une coordination synchronisée lors du bond ou de la fuite. Une telle complexité des démarches est particulièrement exacerbée chez le cheval, qui met en jeu des modes de coordination bilatérale distincts au pas, au trot et au galop. Ces observations soulèvent des questions majeures sur l'organisation du CPG locomoteur dont beaucoup restent à documenter. Existe-t-il des circuits neuronaux dédiés à chaque type de démarche et comment sont-ils sélectionnés? Un déterminisme génétique peut-il expliquer les variations entre espèces?

\section{Génétique du développement} et neurophysiologie

Répondre à ces questions requiert la manipulation sélective de groupes neu- ronaux distincts au sein du CPG locomoteur, une tâche longtemps rendue difficile par la nature enchevêtrée des populations spinales. Les avancées de la génétique du développement ont ouvert de nouvelles voies méthodologiques prometteuses [2, 3]. En particulier, la production des différents types neuronaux apparaît largement codée génétiquement pendant le développement embryonnaire sur la base de l'expression combinatoire de facteurs de transcription au sein des progéniteurs neuraux [4] (Figure 1A). En manipulant ces gènes de développement chez l'animal, il est ainsi possible de visualiser, tracer, ou même éliminer, avec une précision jamais atteinte auparavant, des populations neuronales choisies (dont les neurones individuels ont une origine développementale commune), et de révéler ainsi leur connectivité et leur rôle fonctionnel. Dans cette étude menée par le Professeur Ole Kiehn à Stockholm et en collaboration avec des équipes françaises, nous avons mis en œuvre ces outils pour l'étude des neurones impliqués dans la coordination bilatérale des membres postérieurs lors de la marche spontanée chez la souris [5].

\section{Les neurones commissuraux dits V0} assurent l'alternance bilatérale Chez la souris, l'alternance bilatérale est la démarche prépondérante. Le modèle classique proposait que cette alternance soit le reflet d'une inhibition mutuelle des CPG droit et gauche via des neurones 


\section{A Facteurs de transcription et diversité neuronale}

Domaines organisés de progéniteurs distincts au sein du tube neural embryonnaire

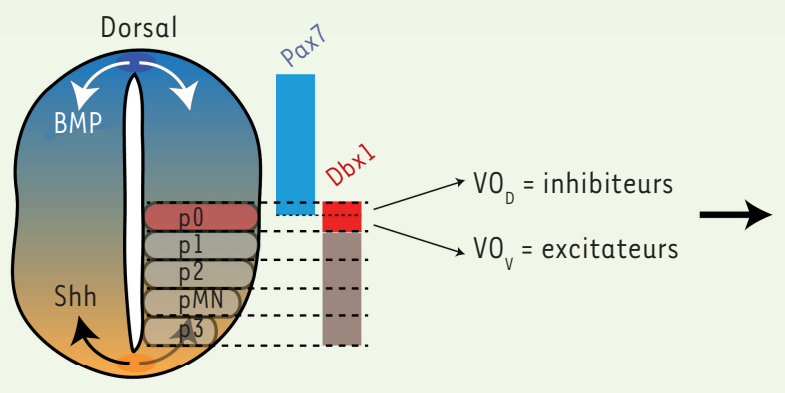

Ventral
Types neuronaux distincts, enchevêtrés au sein de la moelle épinière

\section{B Approche de génétique intersectionelle}

Lignée floxée : Dbx $1^{\text {lox-stop-lox-DTA }}$

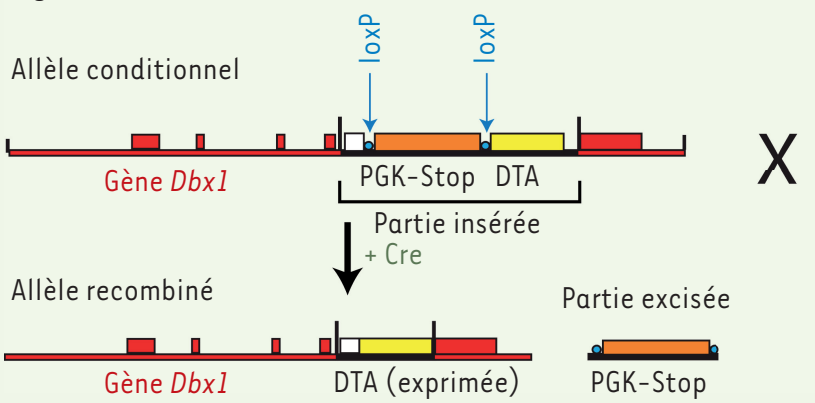

Lignée Cre

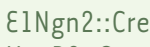

HoxB8::Cre

Pax $7::$ Cre

Vglut2::Cre
Ablation conditionelle ciblée

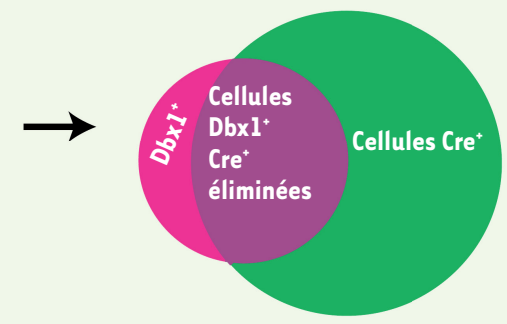

Figure 1. Génétique du développement et génétique intersectionelle chez la souris. A. En réponse à des gradients moléculaires (BMP, Shh), les progéniteurs neuraux s'organisent en domaines caractérisés par l'expression combinatoire de facteurs de transcription. Les progéniteurs exprimant Dbxl définissent le domaine p0, lui-même divisé en sous-domaines ventraux et dorsaux sur la base de l'expression de Pax7. De ces domaines organisés dérivent les différents types cellulaires de la moelle épinière. $B$. Une première lignée de souris porte, au sein du gène $D b x l$, une insertion permettant l'expression conditionnelle d'un allèle d'intérêt, ici la toxine diphtérique A (DTA). Après excision des séquences «stop » par une enzyme (la Cre), la DTA sera exprimée (allèle recombiné) et engendrera l'élimination de la cellule. La Cre, absente du génome murin, est apportée par croisement avec une seconde lignée génétiquement modifiée, autorisant ainsi l'ablation des cellules exprimant à la fois Dbxl et le gène contrôlant l'expression de la Cre.

commissuraux inhibiteurs, c'est-à-dire dont l'axone croise la ligne médiane et fait une synapse inhibitrice du côté controlatéral [1, 2]. Pour autant, ce schéma est longtemps resté hypothétique, et la nature des différentes populations commissurales impliquées dans l'élaboration des différents types de démarches restait peu documentée. En raison de leur positionnement dans la moelle ventrale et de leur nature largement commissurale, nous nous sommes intéressés aux neurones dits V0, dérivés des progéniteurs exprimant le facteur de transcription Dbxl [6] (progéniteurs dits $p 0$, Figure IA). Pour définir leur rôle fonctionnel, nous les avons manipulés par une approche de génétique dite intersectionelle [7] (Figure 1B).
Dans un premier temps, nous avons procédé à l'élimination complète des neurones VO sélectivement dans la moelle épinière, en épargnant ceux du tronc cérébral qui jouent un rôle vital dans la genèse du rythme respiratoire $[8,9]$. Les mutants obtenus respirent donc et présentent un phénotype locomoteur surprenant: s'ils sont bien capables de se déplacer, leurs membres inférieurs droits et gauches, à la différence de ceux des souris sauvages, ne peuvent pas se mouvoir en alternance. Au contraire, ils sont parfaitement synchronisés et l'animal se déplace, quelque soit sa vitesse, à la manière d'un lapin (Figure 2A, B). Ces observations démontrent que l'élaboration de l'alternance bilatérale est impu- table aux seuls neurones commissuraux V0. En leur absence, le CPG locomoteur est uniquement capable d'élaborer des mouvements synchrones, indiquant en outre la présence d'un circuit synchronisateur indépendant des neurones VO.

L'inhibition directe n'explique pas tout Bien que par définition tous dérivés du même groupe de progéniteurs po exprimant Dbxl, les neurones V0 comprennent un contingent inhibiteur et un contingent excitateur [10]. L'hypothèse la plus simple propose que le phénotype locomoteur décrit ci-dessus soit imputable aux Vo inhibiteurs, source d'inhibition réciproque directe; nous avons donc réalisé leur élimination sélective. 
A

Souris sauvage : alternance bilatérale

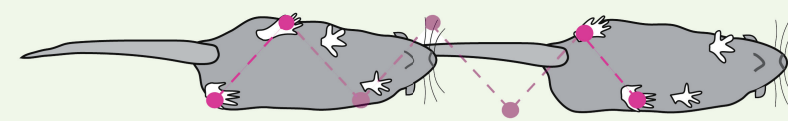

Souris privée de neurones VO : synchronisation bilatérale

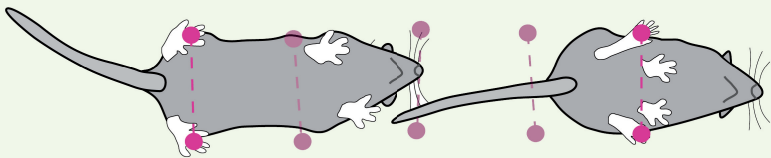

B

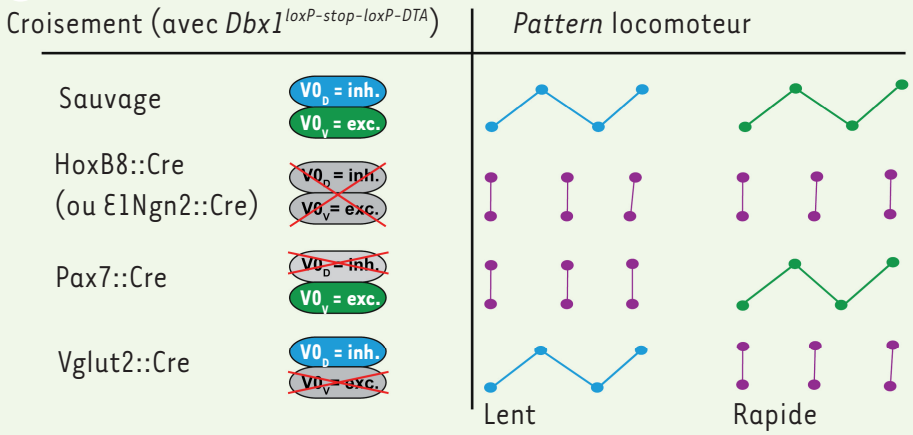

C

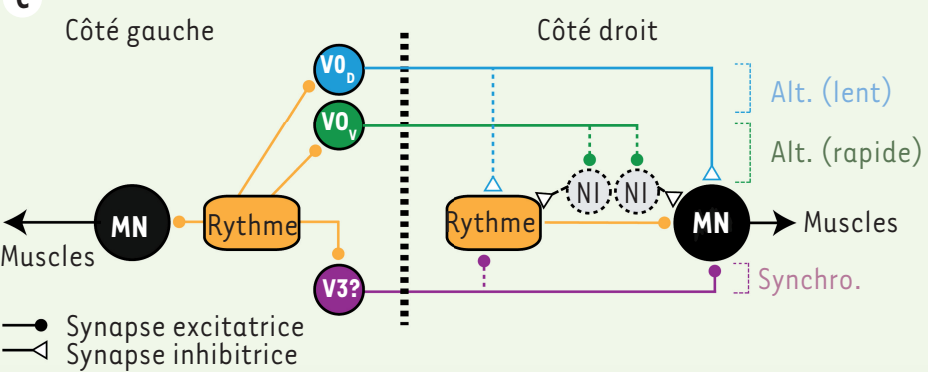

Nous avons tout d'abord montré que les neurones $V 0$ inhibiteurs, dits $V 0_{D}$, sont spécifiquement dérivés du sous-groupe de progéniteurs $\mathrm{Dbxl}^{+}$qui coexpriment également le facteur de transcription Pax7 (Figure 1A). Ainsi, nous avons pu obtenir des animaux sélectivement privés des $V 0_{D}$ inhibiteurs (Figures $1 B, 2 B$ ). Ces mutants, à l'image de ceux décrits ci-dessus, sont incapables de produire des décharges bilatérales alternées des nerfs locomoteurs en conditions normales. En revanche, en augmentant pharmacologiquement la fréquence du rythme locomoteur, nous avons observé que l'activité des racines nerveuses devenait largement alternée. Tout se passe donc comme si ces animaux présentaient une démarche anormalement synchrone aux vitesses les plus lentes, mais retrouvaient une démarche normalement alternée aux vitesses les plus élevées (Figure 2B). Autrement dit, les neurones commissuraux $\mathrm{V}_{\mathrm{D}}$ inhibiteurs sont bien requis aux vitesses lentes et modérées. En revanche, aux vitesses rapides, le réseau est capable de produire, en leur absence, un pattern d'alternance. Quelles sont donc les populations neuronales commissurales susceptibles de prendre le relais à ces vitesses?

\section{Le rôle inattendu des commissures excitatrices}

Les neurones $V 0$ épargnés, les neurones dits $\mathrm{V}_{V}$, apparaissent donc comme candidats à l'élaboration de l'alternance aux vitesses rapides, malgré leur nature largement excitatrice (comme l'illustre
Figure 2. Rôle des neurones Vo dans l'alternance bilatérale. A. L'animal se déplaçant dans un couloir transparent est filmé, et les mouvements des membres inférieurs recueillis et analysés. La perte complète des neurones $\mathrm{V} 0$ entraîne des mouvements anormalement synchronisés. B. Les croisements de la lignée Dbxl loxP-stop-loxP-DTA (voir Figure 1) avec diverses lignées Cre conduisent à l'élimination complète des neurones $v 0$ dans la moelle épinière (HoxB8::Cre), ou ciblée des contingents inhibiteurs (Pax7::Cre) et excitateurs (Vglut2::Cre). Les phénotypes locomoteurs résultants illustrent le rôle de chaque population dans l'alternance bilatérale à différentes vitesses. C. Représentation schématique simplifiée des circuits commissuraux au sein du CPG locomoteur. L'activité rythmique est transmise aux motoneurones (MN) ipsilatéraux ainsi qu'au réseau en charge de la mise en forme ou pattern. L'étude révèle une organisation duelle des circuits qui assurent l'alternance bilatérale : les $v 0_{D}$ inhibiteurs sont requis aux vitesses lentes uniquement, et procurent vraisemblablement l'inhibition controlatérale directe. Les $\mathrm{V}_{\mathrm{V}}$ excitateurs sont recrutés aux fréquences les plus rapides, et procureraient une inhibition indirecte via des interneurones inhibiteurs (NI).

l'expression du transporteur vésiculaire du glutamate Vglut2). Pour le vérifier, nous avons obtenu des animaux privés sélectivement des neurones $\mathrm{V} \mathrm{O}_{\mathrm{V}}$ et analysé les conséquences sur l'élaboration de la démarche locomotrice. Fait remarquable, ces animaux présentent, à la fois in vivo et in vitro, un phénotype locomoteur inverse de celui décrit ci-dessus après l'élimination des $V 0_{D}$ inhibiteurs: les membres inférieurs droits et gauches alternent maintenant aux vitesses lentes et modérées, puis adoptent une coordination bilatérale synchrone lorsque l'animal se déplace plus rapidement (Figure 2B). Ces observations indiquent donc que les commissures $V 0_{V}$ excitatrices, qui ne sont pas requises pour élaborer un pattern d'alternance aux vitesses de locomotion lentes, deviennent indispensables aux vitesses les plus élevées. 


\section{Conclusion}

Ces résultats révèlent un schéma d'organisation nouveau au sein du CPG locomoteur, intégrant mise en forme et vitesse: deux circuits neuronaux distincts, mais partageant une origine génétique commune, assurent l'alternance bilatérale des mouvements locomoteurs en fonction de la vitesse (Figure 2C). Des commissures inhibitrices, les $\mathrm{V}_{\mathrm{D}}$, assurent l'alternance aux vitesses lentes et modérées, vraisemblablement via une inhibition réciproque directe (Figure 2C). Beaucoup plus inattendue est la nécessité exclusive des commissures excitatrices $\mathrm{VO}_{\mathrm{V}}$ aux vitesses les plus élevées de locomotion. Ces neurones procureraient leur inhibition controlatérale indirectement, via des interneurones inhibiteurs dont la nature reste à identifier. L'ajout d'une synapse procure vraisemblablement une amplification du signal, nécessaire pour fournir, in fine, une inhibition controlatérale efficace lorsque le réseau est très actif, comme c'est le cas lors des déplacements rapides. Enfin, un troisième réseau neuronal, indépendant de Dbxl, assurerait la synchronisation bilatérale lors du saut ou de la fuite. Ainsi, l'adaptabilité du comportement locomoteur à la vitesse de déplacement impliquerait la sélection de circuits neuronaux dédiés. Cette organisation modulaire à l'échelle neuronale pourrait sous-tendre les changements comportementaux à l'échelle de l'organisme: lorsque nous courons, davantage de muscles sont recrutés et la séquence de recrutement est finement adaptée. II sera maintenant intéressant d'étudier les mécanismes qui gouvernent le recrutement différentiel de ces réseaux selon les démarches.

$\varepsilon$ n outre, en attribuant une nature et un rôle distincts aux neurones dérivés des deux sous-domaines de progéniteurs $D b x I^{+}$, cette étude illustre l'impact du déterminisme génétique sur l'élaboration de traits comportementaux. Les mécanismes qui raffinent localement le destin neuronal pour aboutir à la formation des territoires fonctionnels à partir de gènes largement partagés restent maintenant à documenter. Enfin, et au regard de la large conservation phylogénétique des programmes développementaux, des modifications évolutives de la signalisation dépendante de Dbxl peuvent-elles expliquer les divergences de coordination bilatérale chez différentes espèces? Poursuivre ces questions promet des avancées majeures dans la compréhension des origines génétiques et évolutives de la formation et de la complexité du système nerveux dans son ensemble. Ces études constituent également des fondements nécessaires aux interventions thérapeutiques ciblées qui requièrent une connaissance détaillée des bases génétiques et neuronales à l'origine d'un comportement donné. $\diamond$

Neurons in motion: spinal networks controlling left-right alternation of walking movements deciphered by genetics

\section{LIENS D'INTÉRÊT}

Les auteurs déclarent n'avoir aucun lien d'intérêt concernant les données publiées dans cet article.

\section{RÉFÉRENCES}

1. Kiehn 0. Locomotor circuits in the mammalian spinal cord. Annu Rev Neurosci 2006 ; 29 : 279-306.

2. Kiehn 0 . Development and functional organization of spinal locomotor circuits. Curr Opin Neurobiol 2011 ; $21: 100-9$

3. Goulding M. Circuits controlling vertebrate locomotion: moving in a new direction. Nat Rev Neurosci 2009 ; $10: 507-18$.

4. Jessell TM. Neuronal specification in the spinal cord: inductive signals and transcriptional codes. Nat Rev Genet $2000 ; 1: 20-9$.

5. Talpalar, AE, Bouvier J, Borgius L, et al. Dual-mode operation of neuronal networks involved in left-right alternation. Nature $2013 ; 500: 85-8$.

6. Pierani A, Moran-Rivard L, Sunshine MJ, et al. Control of interneuron fate in the developing spinal cord by the progenitor homeodomain protein Dbxl. Neuron $2001 ; 29: 367-84$.

7. Bielle F, Griveau A, Narboux-Neme N, et al. Multiple origins of Cajal-Retzius cells at the borders of the developing pallium. Nat Neurosci 2005 ; 8 : 1002-12.

8. Bouvier J, Thoby-Brisson M, Renier N, et al. Hindbrain interneurons and axon guidance signaling critical for breathing. Nat Neurosci $2010 ; 13$ : 1066-74.

9. Viemari JC, Menuet C, Hilaire G. Complexe de préBötzinger et automatisme respiratoire. Med Sci (Paris) $2013 ; 29: 875-82$.

10. Lanuza GM, Gosgnach S, Pierani A, et al. Genetic identification of spinal interneurons that coordinate left-right locomotor activity necessary for walking movements. Neuron 2004 ; 42 : 375-86.

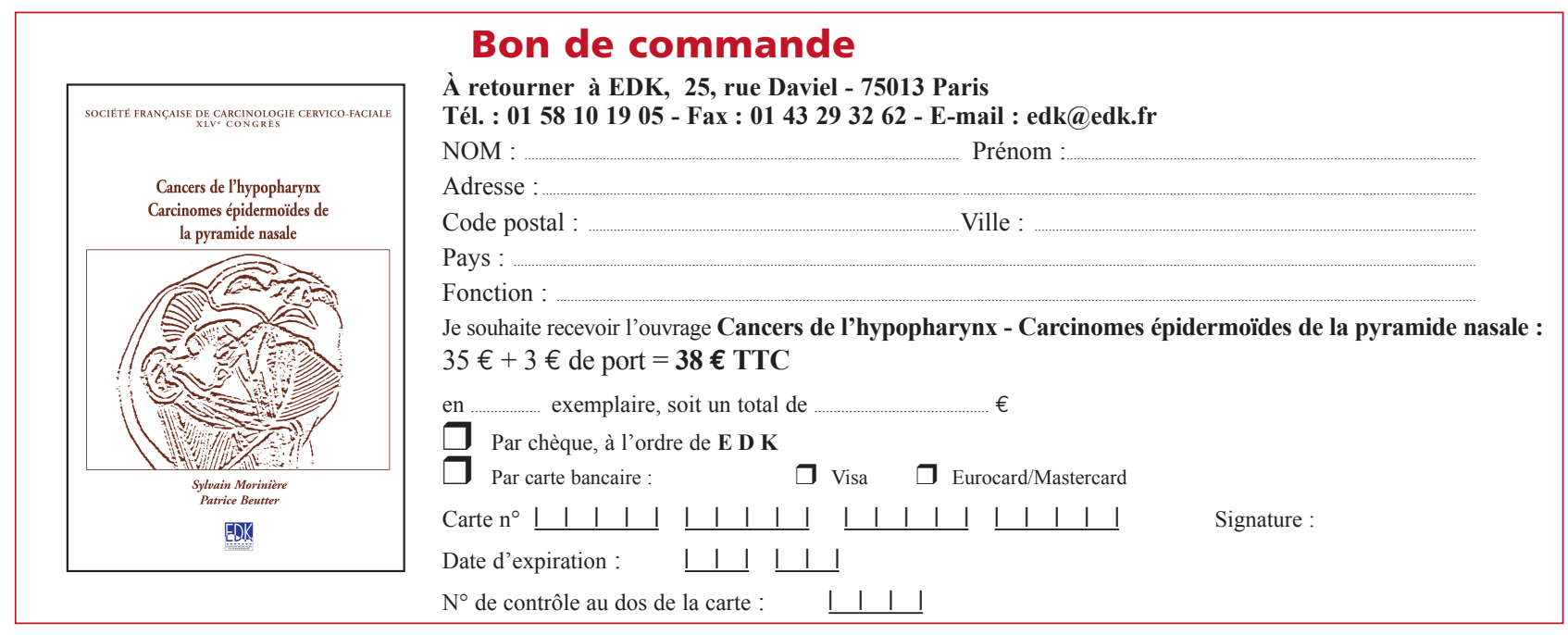

